\title{
PARTICIPANTS LIST
}

Afanasev, Andrei

Hampton University

afanas@jlab.org

Physics Department

Hampton University

Hampton, VA 23668

Ahmad, Saeed

Univ of Virginia

sa8y@virginia.edu

Physics (Univ of Virginia)

382 McCormick Road

Charlottesville, VA 22904 USA

Alexandrou, Constantia

University of Cyprus

alexand@ucy.ac.cy

Department of Physics

P.O. Box 20537

Nicosia, 1678 Cyprus

Ashery, Daniel

Tel Aviv University

ashery@tauphy.tau.ac.il

Ramat Aviv

Tel Aviv, Israel

Avagyan, Harut

JLab

avakian@jlab.org

Baldini, Rinaldo

INFN-LabNazFrascati baldini@lnf.infn.it

Roma, 00186 ITALY

Balewski, Jan

IUCF

jtbalews@indiana.edu

Bloomington, IN 47408

Bedir, Islam

ODU

bedir@physics.odu.edu

12000 Jefferson Avenue,

CEBAF Center

Newport News, VA 23606

Braun, Vladimir

Regensburg University

vladimir.braun@physik.uni-

regensburg.de

Universitaet Regensburg, Institut

fuer Theor.Physik,

Universitaetsstrasse 31

Regensburg, D-93053 Germany

Brodsky, Stanley

SLAC, Stanford University

sjbth@slac.stanford.edu

2575 Sand Hill Road

Menlo Park, CA 94025

Burkardt, Matthias

NMSU

burkardt@nmsu.edu 
Department of Physics, MSC 3D

New Mexico State University

Las Cruces, NM 88011

Burkert, Volker

Jefferson Lab

burkert@jlab.org

12000 Jefferson Avenue

Newport News, VA 23606

Camsonne, Alexandre

Jefferson Laboratory

camsonne@jlab.org

12000 Jefferson Avenue

Cebaf Center A120

Newport News, VA 23606

Canan, Mustafa

Old Dominion University

mcanan@odu.edu

Cardman, Lawrence

JLab

cardman@jlab.org

12000 Jefferson Ave

Newport News, VA 23606

Carlson, Carl

William \& Mary

carlson@physics.wm.edu

Chen, Jian-ping

Jefferson Lab

jpchen@jlab.org

Chirilli, Giovanni

JLAB/ODU

chirilli@jlab.org

4600 Elkhorn ave.

Norfolk, VA 23529

Christy, Michael

Hampton University christy@jlab.org

Hampton, VA 23668

Chudakov, Eugene

JLab

gen@jlab.org

12000 Jeffereson ave

Newport News, VA 23606

Commisso, Melissa

UVA

mac5cx@virginia.edu

D'Hose, Nicole

CEA- Scalay

nicole.dhose@cea.fr

Dalton, Mark

University of the Witwatersrand

dalton@jlab.org

School of Physics

Private Bag 3

Johannesburg, 2192 South Africa

Danagoulian, Samuel

North Carolina A\&T

State University

danagu@jlab.org

Department of Physics

1601 E. Market St.

Greensboro, NC 27411

Day, Donal

University of Virginia

dbd@virginia.edu

Department of Physics

McCormick Road

Charlottesville, VA 22903

de Jager, Kees

Jefferson Lab

kees@jlab.org

12000 Jefferson Avenue

Newport News, VA 23606 
Dubnicka, Stanislav

SAS

fyzidubn@savba.sk

Inst.of Physics, SAS, Bratislava

Dubravska cesta

Bratislava, SK 84311

Slovak Republic

Dubnickova, Anna

Comenius University

dubnickova@fmph.uniba.sk

Dept.of Theor.Phys., FMFI UK,

Mlynska dolina

Bratislava, SK 84245

Slovak Republic

Ellinghaus, Frank

University of Colorado

Frank.Ellinghaus@desy.de

Brookhaven National Lab,

Physics Department

Building $510 \mathrm{C}$

Upton, NY 11973

Elouadrhiri, Latifa

Jefferson Lab

latifa@jlab.org

Ent, Rolf

Jefferson Lab

lcarlson@jlab.org

Feuerbach, Robert

College of William and Mary

feuerbac@jlab.org

Gamberg, Leonard

Penn State Berks

lpg10@psu.edu

Reading, PA 19610

Gaskell, Dave

JLab

gaskelld@jlab.org
Gilfoyle, Gerard

University of Richmond

gilfoyle@jlab.org

Physics Department,

University of Richmond

28 Westhampton Way

Richmond, VA 23173

Gilman, Ronald

Rutgers/JLab

gilman@jlab.org

Girod, Francois-Xavier

Jefferson Lab

fxgirod@jlab.org

Goldstein, Gary

Tufts University

gary.goldstein@tufts.edu

Department of Physics

Tufts University

Medford, MA 02155

Gothe, Ralf

University of South Carolina

gothe@sc.edu

Department of Physics

and Astronomy

712 Main Street

Columbia, SC 29208

Griffioen, Keith

College of William and Mary

griff@jlab.org

Grosse Perdekamp, Matthias

UIUC and RBRC

mgp@uiuc.edu

1110 W. Green Str.

Urbana, IL

Guidal, Michel

IPN Orsay 
guidal@ipno.in2p3.fr

rue Georges Clemenceau

Orsay, 91405 France

Hafidi, Kawtar

Argonne National Laboratory kawtar@anl.gov

9700 S Caa Av

Argonne, IL 60439

Hansen, Ole

Jefferson Lab

ole@jlab.org

12000 Jefferson Avenue

Suite 4

Newport News, VA 23606

Hayrapetyan, Avetik

University of Michigan

Avetik.Airapetian@desy.de

HERMES/DESY

Notkestrasse 85

Hamburg, 22607 Germany

Honkanen, Heli

UVa

hh9e@virginia.edu

Horn, Tanja

JLab

hornt@jlab.org

Hoyer, Paul

University of Helsinki

paul.hoyer@helsinki.fi

Hwang, Dae Sung

Sejong University

dshwang@sejong.ac.kr

Gwangjin-Gu, Gunja-Dong 98

Seoul, 143-747 South Korea

Hyde, Charles

Old Dominion University and
University Blaise Pascal

hyde@jlab.org

Laboratoire de Physique

Corpusculaire CNRS/IN2P3

24 avenue des Landais

AUBIERE, cedex 63177 France

Itard, Florian

University of Blaise Pascal

flosourcier@yahoo.fr

Jain, Pankaj

IIT Kanpur

pkjain@iitk.ac.in

Physics Department

IIT

Kanpur, UP 208016 India

Ji, Chueng

North Carolina State University

ji@ncsu.edu

Dept of Physics

North Carolina State University

Raleigh, NC 27695-8202

Jiang, Xiaodong

Rutgers University

jiang@jlab.org

Jones, Mark

JLab

jones@jlab.org

Khetarpal, Puneet

Rensselaer Polytechnic Institute puneetk@jlab.org

JR Science Center Room 1C25

110 Eighth Street

Troy, NY 12180

Kroll, Peter

Universitaet Wuppertal

kroll@physik.uni-wuppertal.de 
Universitaet Wuppertal

Physik

Wuppertal, D-42097 Germany

Kubarovsky, Valery

$\mathrm{RPI} / \mathrm{Jlab}$

vpk@jlab.org

Jefferson Lab

12000 Jefferson av., suite 5, B105

Newport News, VA 23606

Kuchina, Elena

Rutgers University

kuchina@jlab.org

Kwee, Herry

Arizona State University

herry.kwee@asu.edu

Physics Department,

Bateman Physical Science F-Wing

Arizona State University

Tempe, AZ 85287

Lachniet, Jeffrey

ODU

lachniet@physics.odu.edu

Dept. of Physics

Old Dominion University

Norfolk, VA 23529

Laget, Jean-Marc

JLab

laget@jlab.org

Lansberg, Jean-Philippe

Ecole Polytechnique

Jean-Philippe.Lansberg@

cpht.polytechnique.fr

Ecole Polytechnique - CPHT

Palaiseau, 91128 France

LeRose, John

JLab lerose@jlab.org

JLab 12000 Jefferson Ave

Suite 4, MS 12H3

Newport News, Va 23188

Liuti, Simonetta

University of Virginia

sl4y@virginia.edu

382, McCormick Rd.

Charlottesville, VA 22904-4714

Matevosyan, Hrayr

JLab

hrayr@jlab.org

Theory Center, MS-12H2, Suite 1

12000 Jefferson Avenue

Newport News, VA 23606

Mazouz, Malek

Faculte des sciences de Monastir mazouz@jlab.org

Departement de physique

Monastir, 5000 Tunisia

Mbianda Njencheu, Georgie

Wits University/Jlab

mbianda@jlab.org

School of Physics, Private Bag 3,

Wits University

Johannesburg, Gauteng 2050

South Africa

Miller, Gerald

Physics Dep't UW

miller@phys.washington.edu

University of Washington,

Physics Dep't

Box 351560

Seattle, WA 98195-1560

Mueller, Dieter

University Bochum 
dieter.mueller@physik.uniregensburg.de

Inst. f. Theoretische Physik II

University Bochum

Bochum, 44780 Germany

Nathan, Alan

University of Illinois

a-nathan@uiuc.edu

Department of Physics

1110 W. Green Street

Urbana, IL 61821

Niyazov, Rustam

JLAB

rustam@jlab.org

B120 12000 Jefferson Ave

Newport News, VA 23606

Pentchev, Lubomir

The College of William and Mary

pentchev@jlab.org

Jefferson Lab, 12000 Jefferson Ave. 12H5/F318

Newport News, VA 23606

Perdrisat, Charles

William and Mary

perdrisa@jlab.org

College of William and Mary

Williamsburg, VA 23187

Perez-Benito, Roberto

DESY Hermes

perez@mail.desy.de

Peters, Andreas

Regensburg University

andreas.peters@physik.uni-

regensburg.de

Universitaetsstrasse 31,

Regensburg, Bavaria

93053 Germany
Petratos, Makis

Kent State University

gpetrato@kent.edu

Physics Department

Kent State University

Kent, OH 44236

Psaker, Ales

Hampton University/JLab

apsaker@physics.odu.edu

Punjabi, Vina

Norfolk State University

punjabi@jlab.org

Physics Department

Norfolk State University

Norfolk, VA 23504

Quinn, Brian

Carnegie Mellon University

bquinn@cmu.edu

Pittsburgh, PA 15213

Radyushkin, Anatoly

ODU/JLab

radyush@jlab.org

12000 Jefferson Ave

MS-12H2, Suite 1

Newport News, VA 23606

Richards, David

Jefferson Lab

dgr@jlab.org

Roberts, Craig

Argonne National Laboratory

cdroberts@anl.gov

Physics Division

Argonne National Laboratory

Argonne, IL 60439

Roche, Julie

Ohio University 
rochej@ohio.edu

206 Accelerator Laboratory

Department of Physics

\& Astronomy Athens, OH 45701

Rohrwild, Jurgen

Universitat Regensburg

juergen.rohrwild@physik.uni-r.de

Institut fur theoretische Physik

Regensburg, 93040 Germany

Rostomyan, Armine

DESY

armine.rostomyan@desy.de

Notkestrasse 85

Hamburg, 22607 Germany

Sabatie, Franck

CEA Saclay

fsabatie@cea.fr

Dapnia/SPhN

Gif sur Yvette, 91191 France

Saha, Arun

JLAB

saha@jlab.org

Sandorfi, Andrew

Brookhaven National Laboratory

sandorfi@bnl.gov

Physics Department

Building 510

Upton, NY 11973

Santoro, Joseph

CUA/JLab

santoro@jlab.org

12000 Jefferson Ave

Newport News, VA 23602

Sargsian, Misak

Florida International University

sargsian@fiu.edu
Department of Physics

Florida International University

Miami, FL 33199

Schierholz, Gerrit

DESY

Gerrit.Schierholz@desy.de

Schlegel, Marc

Jefferson Lab

schlegel@jlab.org

Theory Center, MS $12 \mathrm{H} 2$

12000 Jefferson Avenue

Newport News, VA 23606

Seth, Kamal

Northwestern University

kseth@northwestern.edu

Physics Department,

Northwestern University

Evanston, IL 60201

Smith, Elton

JLab

elton@jlab.org

12000 Jefferson Ave

Newport News, Va 23606

Stoler, Paul

RPI

stoler@rpi.edu

Physics Dept

RPI

Troy, NY 12180

Taneja, Swadhin

Ecole Polytechnique

Swadhin.Taneja@

cpht.polytechnique.fr

CPHT, Ecole Polytechnique

Palaiseau, 91128 France

Thomas, Anthony

JLab 
awthomas@jlab.org

Theory Center, MS-12H2, Suite 1

12000 Jefferson Avenue

Newport News, VA 23606

van der Nat, Paul

NIKHEF

travel@nikhef.nl

Kruislaan 409

AMSTERDAM, 1098 SJ

Netherlands

Vanderhaeghen, Marc

William \&Mary/JLab

marcvdh@jlab.org

College of William and Mary

Dept Physics

PO Box 8795

Williamsburg, VA 23187-8795

Villano, Anthony

RPI

villaa@jlab.org
Weiss, Christian

Jefferson Lab

weiss@jlab.org

Theory Center

Jefferson Lab

Newport News, VA 23606

Wojtsekhowski, Bogdan

TJNAF

bogdanw@jlab.org

Yi, Kai

SLAC

yik@slac.stanford.edu

2575 sand hill road

Menlo Park, Ca 94025

Yuan, Feng

Brookhaven National Lab

fyuan@quark.phy.bnl.gov

RIKEN/BNL Research Center

Brookhaven National Laboratory

Upton, NY 11973 